\title{
Evaluation of tubal and peritoneal factors in chlamydia positive infertile women by laparoscope
}

\author{
N Begum ${ }^{1}$, F Hussain ${ }^{1}$, F Deeba $^{2}$, S A Anwary ${ }^{3}$, P Sultana ${ }^{4}$, J Banu $^{5}$
}

\begin{abstract}
Background: Abnormalities or damage to the fallopian tube interferes with fertility and is responsible for abnormal implantation (eg, ectopic pregnancy). Obstruction of the distal end of the fallopian tubes results in accumulation of the normally secreted tubal fluid, creating distention of the tube with subsequent damage of the epithelial cilia (hydrosalpinx). Genital Chlamydia trachomatis infection has a worldwide distribution6 and is now recognized as the single most common cause of tubal peritoneal damage. The study explores the relationship between serum chlamydia antibody titres (CATs) and detection of tubal damage in infertile women. Objective: To Evaluation of tubal and peritonial factors in chlamydia positive infertile women by laparoscope. Methodology: The tubal status and pelvic findings in 138 women underwent laparoscopy for infertility were related to CAT, which was measured using the whole-cell inclusion immunofluorescence test. RESULTS: A total of 138 infertile women who underwent laparoscopic investigation for infertility were identified and they were divided in two groups, on the bsis of presence is absence Chlamydia positive ( $\mathrm{n}=69)$ and Chlamydia Negative $(n=69)$. Demographic status were almost similar between two groups, however service holder was found significantly higher in Chlamydia positive group (17 vs. 7).

Tubal block was found in 44(63.7\%) in Chlamydia positive and 37(53.6\%) in Chlamydia negative. The difference was statistically significant $(p<0.01)$ between two groups. Site of block \& hydrosalpinges was almost similar between two groups. POD was completely obliterated in $10(14.5 \%)$ in Chlamydia positive and $3(4.3 \%)$ in Chlamydia negative. The difference was statistically significant $(\mathrm{p}<0.05)$ between two groups.

Conclusion: Chlamydia serology is useful mainly as a screening test for the likelihood of tubal damage in infertile women and may facilitate decisions on which women should proceed with further investigations without delay. [J Shaheed Suhrawardy Med Coll 2015;5(2): 54-58]
\end{abstract}

Keywords: infertility, Chlamydia serology

Received: April 2013; Revised: June 2015; Accepted: October 2013

\section{Introduction}

Infertility is the failure to conceive (regardless of cause) after 1 year of unprotected intercourse. Infertility affects approximately $10-15 \%$ of reproductive-aged couples ${ }^{1}$. Infertility is caused by male and/or female factors. Male and female factors each account for approximately $35 \%$ of cases. Often, there is more than one factor, with male and female factors combined causing $20 \%$ of infertility. In the remaining $10 \%$ of cases, the etiology is unknown. ${ }^{2}$
The fallopian tubes play an important role in reproduction. After ovulation, the fimbriae pick up the oocyte from the peritoneal fluid that has accumulated in the cul-de-sac. The epithelial cilia transport the oocyte up to the ampulla. The capacitated spermatozoa are transported from the endometrium through the cornual section and advanced through the fallopian tube down into the ampulla, where fertilization occurs. The embryo initiates its early cleaving stages and is propelled upward to arrive at the endometrial

1. DR. Nurjahan Begum, MBBS, MCPS, FCPS (Obs. Gyn), Assistant Professor, Department of Obstetrics and Gynaecology,, Bangabandhu Seikh Mujib Medical University, Dhaka, Bangladesh

1. Dr. Fawzia Hussain, MBBS, MRCOG, MS (Obs. Gyn), Associate Professor, Department of Obstetrics and Gynaecology, Bangabandhu Seikh Mujib Medical University, Dhaka, Bangladesh

2. Dr. Farzana Deeba, MBBS, FCPS, MS (Obs. Gyn), Assistant Professor, Department of Obstetrics and Gynaecology, Bangabandhu Seikh Mujib Medical University, Dhaka, Bangladesh

3. Dr. Shaheen Ara Anwary, MBBS, FCPS, MS (Obs. Gyn), Assistant Professor, Department of Obstetrics and Gynaecology, Bangabandhu Seikh Mujib Medical University, Dhaka, Bangladesh

4. Dr. Parveen Sultana,MBBS, DGO, MCPS (Obs. Gyn), Department of Obstetrics and Gynaecology, Bangabandhu Seikh Mujib Medical University

5. Dr.Jesmin Banu

\section{Correspondence}

DR. Nurjahan Begum, MBBS, MCPS, FCPS (Obs. Gyn), Assistant Professor, Department of Obstetrics and Gynaecology, Bangabandhu Seikh Mujib Medical University, Dhaka, Bangladesh, Phone:+8801199018494,Email: deeba_51@yahoo.com 
cavity at the blastocyst stage (i.e., 96-120 h after ovulation). Abnormalities or damage to the fallopian tube interferes with fertility and is responsible for abnormal implantation (eg, ectopic pregnancy). Obstruction of the distal end of the fallopian tubes results in accumulation of the normally secreted tubal fluid, creating distention of the tube with subsequent damage of the epithelial cilia (hydrosalpinx). Other tubal factors associated with infertility are either congenital or acquired. Congenital absence of the fallopian tubes can be due to spontaneous torsion in utero followed by necrosis and reabsorption. Elective tubal ligation and salpingectomy are acquired causes.

Chlamydia trachomatis is a gram-negative bacterium that infects the columnar epithelium of the cervix, urethra, and rectum, as well as nongenital sites. The bacterium is the cause of the most frequently reported sexually transmitted disease in the United States ${ }^{3}$ Genitourinary infection affects primarily young adults and persons with multiple sex partners. ${ }^{4}$ Women carry a disproportionate burden: CDC statistics show that the overall rate of infection was almost three times higher among women than men ${ }^{5}$.

Genital Chlamydia trachomatis infection has a worldwide distribution $^{6}$ and is now recognized as the single most common cause of tubal peritoneal damage ${ }^{7-8}$.

Infection with $\mathrm{C}$. trachomatis results in the formation of antibodies detectable in serum. In contrast to laparoscopy or HSG, detecting evidence of past chlamydial infection using serology is non-invasive, simple and quick to perform ${ }^{9}$. As such, chlamydia serology may be used as a screening test for tubal damage in infertile women.

This study was done to determine the tubal factors by laparoscopy in chlamydia positive in infertile women.

Objective: To Evaluation of tubal and peritoneal factors in chlamydia positive infertile women by laparoscope.

\section{Materials and Methods}

This cross sectional study was carried out in the Department of Infertility Centre, Bangabandhu Sheikh Mujib Medical University, Dhaka, from July 2009 to June 2011. Prior to the commencement of this study, the research protocol was approved by the Local ethical committee. The objectives of the study along with its procedure, alternative diagnostic methods, risks and benefits of this study were explained to the patients in easily understandable local language and then informed consent was taken from each patient. It was assured that all information and records would be kept confidential and the procedure would be helpful for both the physician and the patients in making rational approach of the patient management. Clinically suspected 138 cases of infertile couple from OPD or inpatient department purposively selected referred above department.

In this cross-sectional study, all women included had a diagnostic laparoscopy for assessment of tubal patency, fibrosis, distortion, or the presence of endometriosis or pelvic adhesions. Women who had a distinct cause of infertility such as ovulatory dysfunction with no index of pelvic disease would not have had a routine laparoscopy and some others conceived before laparoscopy was necessary or arranged. Although different clinicians carried out the laparoscopies over this period of time, each clinician employed the same technique because they were supervised initially by one of two consultants prior to being allowed to assess the pelvis independently. All the clinicians were accredited specialists or senior trainees. Findings were recorded in a standardized way.

Women with tubal damage (or pelvic adhesions not due to endometriosis) served as the 'cases' to be identified by the test (chlamydia serology) and women without damage served as 'controls' regardless of their other infertility diagnoses.

Women were also categorized according to three main findings at laparoscopy: (i) tubal damage; (ii) endometriosis; or (iii) normal pelvis. Tubal damage was diagnosed by the finding of tubal occlusion, and/or distortion of the fimbriae, and/or restrictive tubal ovarian adhesions, in the absence of visible endometriosis.

Severe tubal damage was classified using the 'Hull and Rutherford' classification for tubal disease ${ }^{9-10}$. This classification referred to women with bilateral tubal damage with extensive tubal fibrosis, and/or tubal distension $>1.5$ $\mathrm{cm}$, and/or an abnormal tubal mucosal appearance and/or bipolar occlusion, and/or extensive dense pelvic adhesions.

\section{Laboratory Procedures:}

A clotted blood sample was obtained from the patient prior to the laparoscopy and sent to the Public Health Laboratory Service in Bristol for assay. Both clinical and laboratory personnel were blind as to the pelvic status of the woman at that time. Serum samples were assayed for chlamydia IgG antibody employing the single-antigen inclusion test using indirect immunofluorescence, as previously described by Richmond and Caul11, otherwise known as the whole-cell inclusion immunofluorescence (WIF) assay. This was applied in practice $^{12}$ and modified using C.trachomatis L2 serotype $^{13}$ as antigen to infect McCoy cell monolayers and anti-human IgM-IgA-IgG-fluoresce in conjugate. Dilutions of sera were expressed as antibody titres from 1:64 to $>1: 4096$, or negative $(<1: 64)^{14}$.

\section{Statistical analysis:}

Statistical analyses was carried out by using the Statistical Package for Social Sciences version 20.0 for Windows (SPSS Inc., Chicago, Illinois, USA). A descriptive analysis was performed for all data. The mean values was calculated for continuous variables. The quantitative and qualitative observations were indicated by frequencies and percentages. Chi-Square test was used to analyze the categorical variables was shown with cross tabulation and unpaired t- test was used to analyze the continuous variable was expressed as mean $( \pm \mathrm{SD})$. A P-value will considered to be statistically non significant if $>0.05$ and statistically significant if $\leq 0.05$. 


\section{Results:}

A total of 138 infertile women who underwent laparoscopic investigation for infertility were identified. Complete data including chlamydia serology were available for all cases and subsequent analysis is based on these. The patients were divided in two groups, which were Chlamydia positive $(n=69)$ and Chlamydia Negative $(\mathrm{n}=69)$.

The mean age was found $28.6 \pm 4.7$ years varied from $21-$ 41 years in Chlamydia positive and $27.2 \pm 4.1$ years varied from $20-40$ years in Chlamydia negative. Husband occupational status, educational status, wife educational status, religion and socioeconomic status were almost similar between two groups. On the other hand service holder was found significantly higher in Chlamydia positive group (17 vs. 7). The mean duration of marriage was 7.9 \pm 3.8 years varied from 2- 20 years in Chlamydia positive and $7.2 \pm 4.0$ years varied from 2- 20 years in Chlamydia negative. The mean duration of marriage and duration of subfertility were almost similar between two groups.

Mild dysmenomhoea was found $27(87.0 \%)$ in Chlamydia positive and $24(85.7 \%)$ in Chlamydia negative. Moderate dysmenomhoea was $1(3.2 \%)$ in Chlamydia positive but not found in Chlamydia negative patients. Severe dysmenomhoea was $3(9.7 \%)$ in Chlamydia positive and $4(14.3 \%)$ in Chlamydia negative. The difference was not statistically significant $(\mathrm{p}>0.05)$ between two groups.

Tubal block was found in $44(63.7 \%)$ in Chlamydia positive and $37(53.6 \%)$ in Chlamydia negative. The difference was statistically significant $(p<0.01)$ between two groups. Site of block \& hydrosalpinges was almost similar between two groups. Partially POD was found $11(15.9 \%)$ in Chlamydia positive and $6(8.7 \%)$ in Chlamydia negative. Completely $10(14.5 \%)$ in Chlamydia positive and $3(4.3 \%)$ in Chlamydia negative. The difference was statistically significant $(p<0.05)$ between two groups.

Table I: Distribution of the study patients by type of infertility $(\mathbf{n}=138)$

\begin{tabular}{lccccc}
\hline $\begin{array}{l}\text { Type of } \\
\text { infertility }\end{array}$ & \multicolumn{2}{c}{$\begin{array}{c}\text { Chlamydia Positive } \\
(\mathbf{n}=\mathbf{6 9})\end{array}$} & $\begin{array}{c}\text { Chlamydia Negative } \\
(\mathbf{n = 6 9 )}\end{array}$ & p value \\
\cline { 2 - 6 } & $\mathrm{n}$ & $\%$ & $\mathrm{n}$ & $\%$ & \\
\hline Primary & 50 & 72.5 & 51 & 73.9 & \\
Secondary & 19 & 27.5 & 18 & 26.1 & $0.847^{\mathrm{ns}}$ \\
\hline
\end{tabular}

$\mathrm{ns}=$ not significant, $\mathrm{P}$ value reached from chi square test

Table II: Distribution of the study patients by tubal block $(\mathrm{n}=138)$

\begin{tabular}{lccccc}
\hline Tubal block & \multicolumn{2}{c}{$\begin{array}{c}\text { Chlamydia Positive } \\
(\mathbf{n = 6 9 )}\end{array}$} & $\begin{array}{c}\text { Chlamydia Negative } \\
(\mathbf{n = 6 9 )}\end{array}$ & p value \\
\cline { 2 - 6 } & $\mathrm{n}$ & $\%$ & $\mathrm{n}$ & $\%$ & \\
\hline Tubal block & 44 & 63.7 & 37 & 53.6 & \\
Bilateral & 21 & 30.4 & 11 & 15.9 & $0.01^{\mathrm{s}}$ \\
Unilateral & 23 & 33.3 & 26 & 37.7 & \\
Patent tube & 25 & 36.2 & 32 & 46.4 & \\
\hline
\end{tabular}

Table III: Distribution of the study patients by site of block \& hydrosalpinges $(\mathbf{n}=138)$

\begin{tabular}{lccccc}
\hline Site of block & \multicolumn{2}{c}{$\begin{array}{c}\text { Chlamydia Positive } \\
(\mathbf{n = 6 9 )}\end{array}$} & $\begin{array}{c}\text { Chlamydia Negative } \\
(\mathbf{n = 6 9 )}\end{array}$ & p value \\
\cline { 2 - 6 } & $\mathrm{n}$ & $\%$ & $\mathrm{n}$ & $\%$ & \\
\hline Fimbrial & 27 & 39.1 & 27 & 39.1 & \\
Cornual & 7 & 10.1 & 7 & 10.1 & $0.171^{\mathrm{ns}}$ \\
Block with adhesion & 19 & 27.5 & 19 & 27.5 & \\
& & & & & \\
Hydrosalpinges & 25 & 36.2 & 25 & 36.2 & \multirow{2}{*}{${ }^{\mathrm{n}}$} \\
$\quad$ Unilateral & 16 & 23.2 & 16 & 23.2 & $0.321^{\mathrm{ns}}$ \\
Bilateral & 9 & 13.0 & 9 & 13.0 & \\
Beaded & 20 & 29.0 & 20 & 29.0 & \\
\hline
\end{tabular}

ns=not significant, $\mathrm{P}$ value reached from chi square test

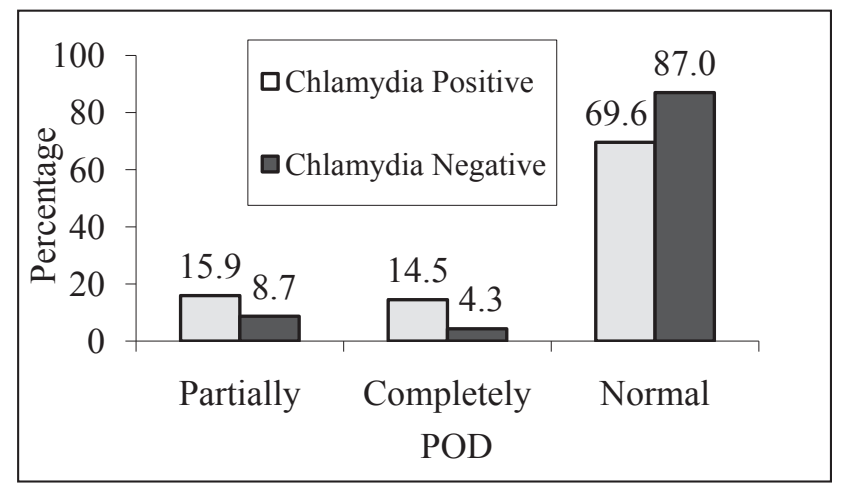

Figure 1: Bar diagram showing obliteration of POD of the patients

\section{Discussion}

This study used laparoscopy on all patients confirms that past infection with C.trachomatis is associated with a significantly increased risk of women suffering tubal infertility, as shown by others 12,15-20. Negative chlamydia serology $(<1: 64)$ does not, however, preclude the diagnosis of tubal damage. Conversely, high titres do not necessarily indicate the presence of tubal damage, as shown by the high titres observed in some women with a normal pelvis in the present study.

Laparoscopy is the accepted gold standard for the diagnosis of tubal damage20. The high prevalence of tubal damage observed may reflect the prolonged duration of infertility (3.8 years) of the women studied. The present study, showed moist of the patients had more than 5 years Nonetheless, a significant proportion of patients who underwent laparoscopy had no pelvic damage and were infertile due to other causes such as sperm or ovulatory dysfunction and unexplained infertility. Tube may be blocked other than Chlamydia microorganism.

The relatively high sero-prevalence of positive CAT and the relatively low proportion of women who give a history of previous PID attest to chlamydial infection being mainly asymptomatic 21.6.

The sensitivity of chlamydia serology in detecting 
tuboperitoneal damage has been demonstrated by others22-24, including a meta-analysis17. However, these studies included women with endometriosis considered as positive cases. Anestad et al. found that pelvic adhesions (not due to endometriosis) were the most frequent sequelae associated with a high CAT. The findings of the study suggested that adhesions were the most likely consequence of chlamydial infection, with occlusion being a manifestation of more severe infection associated with higher titres, consistent with our findings.25 chlamydia and gonorrhoea are both common causes of PID and often co-exist 26.It is therefore plausible that in the women who had a history of acute PID and had negative chlamydia serology, this was caused by gonorrhoea or other organisms 27.

A meta-analysis showed that the performance of chlamydia antibody testing depended on the assay used, and found the WIF test with the enzyme-linked immunosorbent assay (ELISA) and micro-immunofluorescence (MIF) test to be superior to the immunoperoxidase assay 17. However, the studies examined were not strictly comparable because some were based on tubal damage diagnosed by HSG alone, and non-uniform cut-off levels were used. The immunofluorescence test employed in the present study is highly sensitive, as shown by a blinded comparative study of other serological tests for C.trachomatis antibody 14 .

Consequently, women with positive serology but with a normal pelvis may have had non-genital chlamydia infection. In these cases, cross-reactive responses to past infection with other species of chlamydia such as Chlamydia pneumoniae or Chlamydia psittaci 17,21 is a possibility, but difficult to account for. Time-related antibody titre decline is a possible reason for false negatives (i.e. negative serology but positive laparoscopy), but this issue may be controversial.

Because there are justified constraints to the indiscriminate use of laparoscopy and HSG, there is a need to minimize the number of patients subjected to these diagnostic investigations who do not have disease (false positives). If laparoscopy is readily available and the primary aim of screening is to avoid delay in referral for IVF or tubal surgery in those with significant tubal damage, false negatives have to be minimized. As such, a low cut-off may be the preferred option in view of its higher sensitivity. To achieve the objective of identifying a subgroup of infertile women for further investigation, a cut-off level is required. However, a universal single cut-off which splits women into two groups is likely to be controversial.

It is tempting to suggest that early detection of a disease is an end in itself. However, the spectrum of disease varies according to the severity and extent of lesion9. The present study is clear in demonstrating that severe damage is more likely in women with higher titres. This implies that increasing antibody titres are quantitatively related to both the presence of tubal damage and the severity of tubal damage. Thus identification of trivial disease such as minor filmy adhesions or indeed untreatable conditions such as bilateral distended hydrosalpinges are important in terms of prognosis for fertility for different reasons9. Consequently, identifying women who are at sufficiently high risk of having severe tubal damage impairing fertility may be more important than identifying women with minimal tubal damage.

\section{Conclusion}

Striking a balance, in a target population between, on the one hand, the severity of the disorder affecting fertility and the prevalence of disease, and, on the other, the availability, costs, hazards and acceptability of invasive diagnosis is a practical necessity. This study shows that using chlamydia serology for screening provides a useful guide to the risk of tubal damage causing infertility but also exposes certain limitations of this method of screening. However, the choice of cut-off level used for screening would depend on the prevalence of the disease in the target population to which it is applied and whether one wants to identify most cases of women with tubal damage or mainly those with severe damage.

\section{References}

1. Trussell J, Wilson C. Sterility in a population with natural fertility. Popul Stud. 1985; 29: 269-86.

2. Maruani P, Schwartz D. Sterility and fecundability estimation. J Theor Biol. Nov 21 1983; 105(2):211-9.

3. Debekausen YA, Evers JL,Land JA,Stals FS.Chlamydia trachomatis antibody testing is more accurate than hysterosalpingography in predicting tubal factor infertility.Fertil steril 1994; 64:833-7.

4. Debattista J, Timms P,Allan J.Immunopathogenesis of Chlamydia trachomatis infection in women.Fertility and Sterility 2003;79(6); 1273-87. 5. Kodman PH,Article A,SeliE(2004).Evidencebased diagnosis\& management of tubal factor infertility.infertility Obstel Gynecol 16(3)221-9

6. Stamm, W.E. (1999) Chlamydia trachomatis infections of the adult. In Holmes, K.K., Sparling, P.F., Mardh, P.E., Lemon, S.M., Stamm, W.E., Piot, P. and Wasserheit, J.N. (eds), Sexually Transmitted Diseases. McGraw-Hill, New York, pp. 407-432.

7. WHO task force on the prevention and management of infertility (1995) Tubal infertility: serologic relationship to past chlamydial and gonococcal infection. Sex. Transm. Dis., 22, 71-77.

8. ESHRE.Capri Workshop.Infertility revised: the state of ART today and tomorrow.Human Reproduction 1996;11:1776-807.

9. Akande, V. (2002) Tubal pelvic damage: prediction and prognosis. Hum. Fertil.5,S15-S20

10. Rutherford, A.J. and Jenkins, J.M. (2002) Hull and Rutherford classification of infertility. Hum. Fertil., 5, S41-S45.

11. Richmond, S.J. and Caul, E.O. (1975) Fluorescent antibody studies in chlamydial infections. J. Clin. Microbiol., 1, 345-52.

12. Conway, D., Glazener, C.M., Caul, E.O., Hodgson, J., Hull, M.G., Clarke, S.K. and Stirrat, G.M. (1984) Chlamydial serology in fertile and infertile women. Lancet, 1, 191-193.

13. Saikku, P. and Paavonen, J. (1978) Single-antigen immuno- uorescence test for chlamydial antibodies. J. Clin. Microbiol., 8, 119-22.

14. Chernesky, M., Luinstra, K., Sellors, J., Schachter, J., Moncada, J., Caul, O., Paul, I., Mikaelian, L., Toye, B., Paavonen, J. et al. (1998) Can serology diagnose upper genital tract Chlamydia trachomatis infections? Studies on women with pelvic pain, with or without chlamydial plasmid DNA in endometrial biopsy tissue. Sex. Transm. Dis., 25, 14-19. 15. Forsey, J.P., Caul, E.O., Paul, I.D. and Hull, M.G. (1990) Chlamydia trachomatis, tubal disease and the incidence of symptomatic and asymptomatic infection following hysterosalpingography. Hum. Reprod., 5, 444-447. 
16. Thomas, K., Coughlin, L., Mannion, P.T. and Haddad, N.G. (2000) The value of Chlamydia trachomatis antibody testing as part of routine infertility investigations. Hum. Reprod., 15, 1079-1082.

17. Mol, B.W., Dijkman, B., Wertheim, P., Lijmer, J., van der Veen, F. and Bossuyt, P.M. (1997) The accuracy of serum chlamydial antibodies in the diagnosis of tubal pathology: a meta-analysis. Fertil. Steril., 67, 1031-1037. 18. Westrom, L. (1987) Pelvic inflammatory disease: bacteriology and sequelae. Contraception, 36, 111-128.

19. Veenemans, L.M. and van der Linden, P.J. (2002) The value of Chlamydia trachomatis antibody testing in predicting tubal factor infertility. Hum. Reprod., 17, 695-698.

20. RCOG (1998) The Management of Infertility in Tertiary Care. Royal College of Obstetricians and Gynaecologists, London

21. Bjercke, S. and Purvis, K. (1993) Characteristics of women under fertility investigation with $\operatorname{IgA} / \operatorname{IgG}$ seropositivity for Chlamydia trachomatis. Eur. J. Obstet. Gynecol. Reprod. Biol., 51, 157-161. 22. Saraiya, M., Berg, C.J., Kendrick, J.S., Strauss, L.T., Atrash, H.K. and
Ahn, Y.W. (1998) Cigarette smoking as a risk factor for ectopic pregnancy. Am. J. Obstet.Gynecol.,178,493-498

23. Bouyer, J., Coste, J., Shojaei, T., Pouly, J.L., Fernandez, H., Gerbaud, L. and Job-Spira, N. (2003) Risk factors for ectopic pregnancy: a comprehensive analysis based on a large case \pm control, population-based study in France. Am. J. Epidemiol., 157, 185-194.

24. Brunham, R.C., Binns, B., McDowell, J. and Paraskevas, M. (1986) Chlamydia trachomatis infection in women with ectopic pregnancy. Obstet. Gynecol., 67, 722-726.

25. Anestad, G., Lunde, O., Moen, M. and Dalaker, K. (1987) Infertility and chlamydial infection. Fertil. Steril., 48, 787-790.

26. Rice, P.A. and Schachter, J. (1991) Pathogenesis of pelvic inflammatory disease. What are the questions? J. Am. Med. Assoc., 266, 2587-2593. 27. Clausen, H.F., Fedder, J., Drasbek, M., Nielsen, P.K., Toft, B., Ingerslev, H.J., Birkelund, S. and Christiansen, G. (2001) Serological investigation of Mycoplasma genitalium in infertilewomen.Hum.Reprod.,16,1866-1874 